\title{
The interaction of hydrogen chloride with ice surfaces: \\ The effects of grain size, surface roughness, and surface disorder
}

\author{
V. F. McNeill, F.M. Geiger, T. Loerting, B. L. Trout, M. J. Molina \\ Correspondence to MJM at: mimolina@ucsd.edu

\section{Supplementary Online Material}

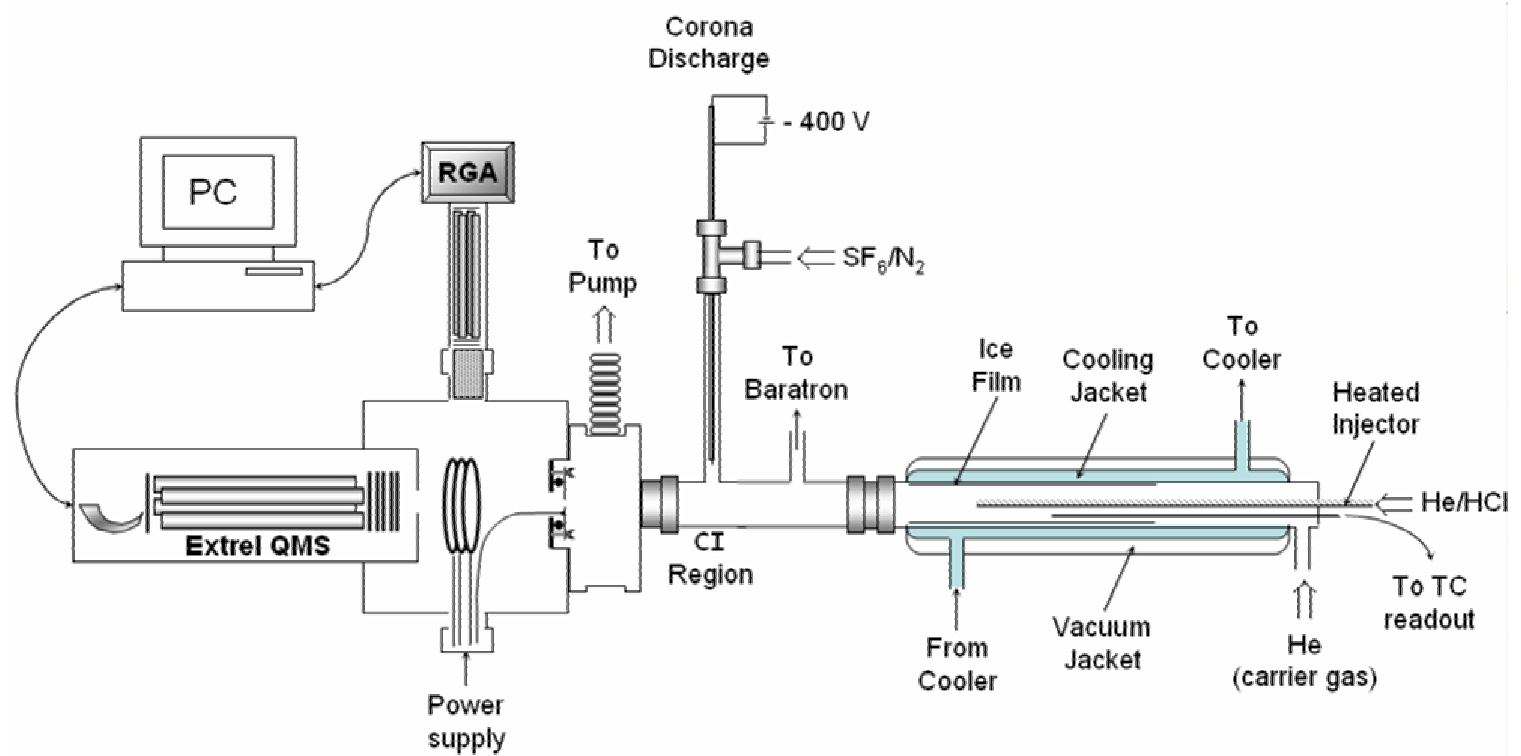

Figure S1. Experimental setup for flow tube-CIMS HCl adsorption experiments.

\section{Ice film preparation}

Smooth ice films formed by freezing from liquid $\mathrm{H}_{2} \mathrm{O}$ were prepared following the method of Abbatt. ${ }^{1}$ The inside of a $35 \mathrm{~cm}$ long glass tube $(2.5 \mathrm{~cm}$ i.d.) was washed with a $5 \%$ solution of $\mathrm{HF}$ in deionized water, thereby creating a wettable glass substrate. After thorough rinsing with HPLC grade water, the inside of the glass insert was wetted with a film of HPLC grade water. The insert was then placed inside the flow tube, which had been pre-cooled to operating temperature, while $\mathrm{He}$ was flowing to maintain an overpressure in the flow tube. Freezing occurred quickly because of the low temperature in the flow tube, yielding a polycrystalline ice film. The ice films formed this way were 
transparent and did not appear frost-like. Since approximately $5 \mathrm{~mL}$ of water was used to prepare each ice film, and each film was approximately $10 \mathrm{~cm}$ long, we estimate the thickness of these films to be several hundreds of microns.

Following literature procedures, ${ }^{2,3}$ we also prepared vapor-deposited ice films on the inside of the glass insert described in the previous section. Briefly, dry He was passed through a water bubbler, and then the saturated stream was introduced to the flow tube maintained at $233 \mathrm{~K}$ via a heated wide-bore $(0.625 \mathrm{~cm}$ o.d.) injector, resulting in a water vapor deposition rate of $3-5 \mathrm{mg} \mathrm{min}^{-1}$. The ice films had a calculated thickness of a few hundred microns based on the flow rate and estimated density. Extra ice was deposited upstream of the ice sample to ensure that the sample was in equilibrium with water vapor, i.e., no net evaporation or condensation took place. For a detailed description of the preparation of zone-refined ice cylinders, please see our previous work. ${ }^{4}$

\section{Flow tube model}

\section{A. Governing equations}

For a dilute solution in laminar flow through a tube:

$$
\frac{\partial C}{\partial t}+2 U\left(1-\left(\frac{r}{R}\right)^{2}\right) \frac{\partial C}{\partial z}=D\left(\frac{1}{r} \frac{\partial}{\partial r}\left(r \frac{\partial C}{\partial r}\right)+\frac{\partial^{2} C}{\partial z^{2}}\right)-R_{l o s s}
$$

where $C$ is the concentration of the solute $(\mathrm{HCl}), U$ is the average velocity, $R$ is the flow tube radius, $D$ is the binary diffusion coefficient for the solute in $\mathrm{He}$ at the conditions of the experiment, and $R_{\text {loss }}$ is the rate of solute loss to the walls (or gas phase reaction). By making the assumption, reasonable for our system, that the characteristic time for diffusion of the solute to the wall is much less than the residence time of a solute molecule within the flow tube, we may take a cross-sectional average of the concentration, thus simplifying eq. 1 , without losing much information:

$$
\frac{\partial \bar{C}}{\partial t}+U \frac{\partial \bar{C}}{\partial z}=D \frac{\partial^{2} \bar{C}}{\partial z^{2}}-R_{l o s s}
$$

where

$$
\bar{C}=\frac{1}{A} \int C d A
$$


The loss term, $R_{\text {loss }}$, may include solute loss to the ice film (surface or bulk) or to gas phase reaction. For surface adsorption, $R_{\text {loss }}$ may be expressed as follows:

$$
R_{\text {loss }}=\frac{2}{R} \frac{d c_{s}}{d t}
$$

where $c_{s}$ is the surface density of $\mathrm{HCl}$ in units of molec $\mathrm{cm}^{-2}$. For absorption into a nearsurface region of thickness $l$ where $l<<R$,

$$
R_{\text {loss }}=\frac{2 l}{R} \frac{\partial C_{s}}{\partial t}
$$

S5.

where $C_{\mathrm{s}}$ is the $\mathrm{HCl}$ concentration on a volume basis in the near-surface region. $C_{\mathrm{s}}$ and $c_{s}$ are described using the models for solute-ice interaction discussed in the following sections.

\section{B. Diffusion through an ice slab}

We accounted for diffusion from the surface layer into the bulk in the gas-surface interaction model as follows: Since diffusion in the ice crystal is slow (the diffusion coefficient, $D_{i} \sim 10^{-12} \mathrm{~cm}^{2} \mathrm{~s}^{-1}$, measured at $258 \mathrm{~K}$ by Thibert and Domine ${ }^{5}$ ), in all cases the characteristic time for diffusion through the ice film, $l^{2} / D_{i}$, is substantially longer than the timescale for the experiment (3600 seconds or less). Therefore, we can model diffusion from the ice surface as diffusion into a semi-infinite slab. ${ }^{6}$ The solution is then

$$
\frac{C_{i}}{C_{s}}=1-\operatorname{erf}\left(\frac{x}{\sqrt{4 D_{i} t}}\right)
$$

S6.

where $C_{i}$ is the $\mathrm{HCl}$ concentration in the ice as a function of distance from the surface, $x$, and erf is the Gaussian error function. The saturation $\mathrm{HCl}$ concentration in single crystals of ice $\left(3.5 \times 10^{-7} \text { moles } \mathrm{cm}^{-3} \text { for adsorption at } 200 \mathrm{~K} \text { and } 10^{-6} \text { Torr } \mathrm{HCl}\right)^{5}$ can serve as an upper bound for $C_{s}$ in the ice crystal for a scenario with surface adsorption but no surface disordering. Figure S2 shows the concentration depth profile at several elapsed times for $\mathrm{HCl}$ diffusing through an ice slab, calculated using eq. 6. One hour after exposure the $\mathrm{HCl}$ is for the most part still confined within the topmost $1 \mu \mathrm{m}$ of the ice film, and the region within $150 \mathrm{~nm}$ of the concentrated surface region has reached within $90 \%$ of the surface concentration via diffusion alone.

The flux of solute into the bulk, J, is derived from eq. 6 as follows: 


$$
J=-D_{i} \frac{\partial C_{i}}{\partial x}=-C_{s} \sqrt{\frac{D_{i}}{\pi t}} .
$$

It is notable that the flux into the bulk is determined by the concentration at the surface. In a scenario in which a thin layer on an ice surface contains a relatively high concentration $C_{s}$ of $\mathrm{HCl}$, diffusive flux from that layer into the bulk could be significant. This is illustrated in Figure S3, which shows flux as a function of time for different values of $C_{s}$ and $D_{i}$. In practical application to our experiments, the large flux at time $t=$ 0 created by the inverse dependence of eq. 7 on time should not be significant because $C_{s}$ will not be constant throughout the experiment (as in this model), but, rather, initially $C_{s}$ $=0$. For a surface layer with $C_{s}=3.5 \times 10^{-7}$ moles $\mathrm{cm}^{-3}$, and $D_{i}=10^{-12} \mathrm{~cm}^{2} \mathrm{~s}^{-1}$, there is a long-term surface flux of between $2 \times 10^{9}$ and $6 \times 10^{9}$ molec $\mathrm{cm}^{-2} \mathrm{~s}^{-1}$, which is established within 500 seconds of the initial exposure and persists through the duration of the experiment. For a surface layer with $C_{s}=10^{-4}$ moles $\mathrm{cm}^{-3}$, this long-term surface flux could be as high as $5 \times 10^{11}$ molec $\mathrm{cm}^{-2} \mathrm{~s}^{-1}$ (which was observed in our previous work for $\mathrm{HCl}$ uptake to zone-refined ice under surface disorder conditions). ${ }^{4}$

\section{Surface Analysis}

One model for surface adsorption is the Langmuir model, based on the concept of a set number of adsorption sites on the surface. The fraction of occupied surface sites, $\theta$, is dimensionless and is related to the surface coverage, $c_{s}$, as follows:

$$
\theta=\frac{c_{s}}{S}
$$

where $S$ is the surface site density. $10^{15}$ molec $\mathrm{cm}^{-2}$ is the value of $S$ widely quoted for ice, although for a polar molecule such as $\mathrm{HCl}$, and a real ice surface with defects, grain boundaries, variable density of dangling - $\mathrm{OH}$ groups, and surface disorder, the definition of an adsorption site becomes less certain. For one adsorbing species, the surface coverage is governed by the following rate equation which describes the balance of molecules adsorbing and desorbing from the surface:

$$
\frac{d \theta}{d t}=k_{a d s} C(1-\theta)-k_{d e s} \theta .
$$

Again, $C$ is the gas phase solute concentration. Inherent in this formulation is the assumption that each adsorbing molecule occupies one site, and that neighboring 
molecules do not interact with one another. At equilibrium, eq. 9 yields the Langmuir isotherm. Additional effects may be incorporated into the surface balance in eq. 9 such as nearest-neighbor interaction, adsorbate disassociation (i.e. occupation of two adsorption sites by each adsorbate molecule), diffusion of the adsorbate on the surface, or incorporation of surface adsorbed solute molecules into the bulk.

In addition to describing surface adsorption and diffusion through the ice bulk, to model this system a mechanism must be assumed for incorporating surface adsorbed $\mathrm{HCl}$ molecules into the bulk. We assumed a constant rate for incorporation, $k_{\text {inc }}$, which is firstorder in $\mathrm{HCl}$ surface coverage. The concentration of $\mathrm{HCl}$ on the ice surface is then governed by

$$
\frac{d c_{s}}{d t}=k_{a d s} \frac{P_{H C l}}{R T}\left(S-c_{s}\right)-k_{d e s} c_{s}-k_{i n c} c_{s}
$$

The near-surface concentration is then described by

$$
\frac{d C_{s}}{d t}=\frac{k_{i n c}}{l} \frac{c_{s}}{S}
$$

where $l$ is the thickness of the near-surface region. Note that, in this model, this depth does not have the same physical significance as in the QLL model, but a near-surface region must be defined in order to determine the near-surface concentration that drives diffusion into the bulk. For these simulations the near-surface region was assumed to have a depth of $100 \mathrm{~nm}$, based loosely on the observations of Rossi and coworkers. ${ }^{7-9}$

\section{D) Numerical solution}

By conceptually dividing the flow tube into a series of well-mixed segments of width $d z$ (see figure $\mathrm{S} 4$ ), we can discretize eqs. 2 and 10 for numerical solution by an explicit finite difference scheme. ${ }^{6,10}$ We can then simultaneously calculate surface, bulk, and gas-phase concentrations as functions of time and space, and ultimately obtain the uptake curve for a variety of cases. All simulations described were programmed and executed using MATLAB 6.0. 


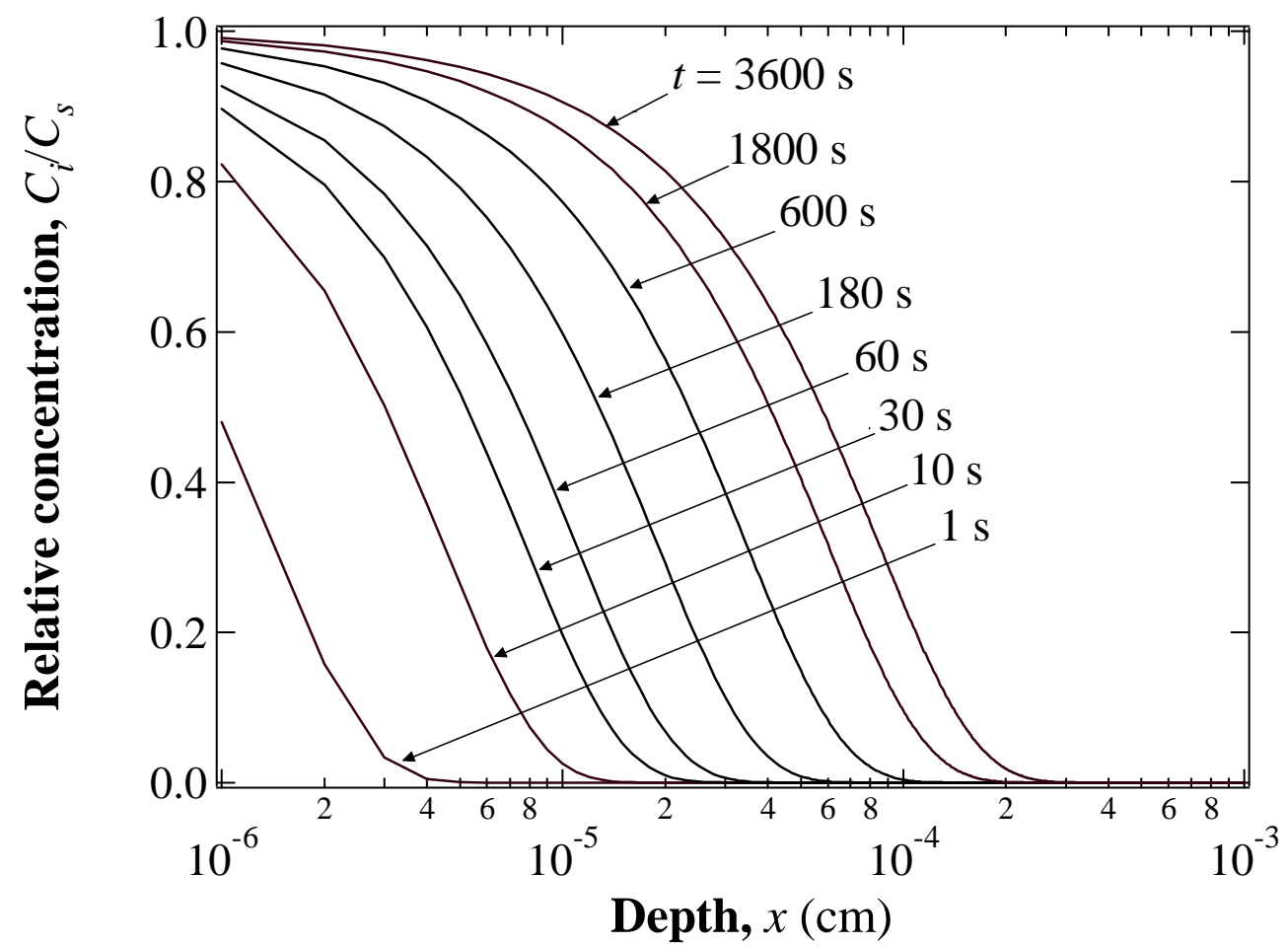

Figure S2. The time evolution of the concentration depth profile on a log scale for $\mathrm{HCl}$ diffusion through an ice slab with constant surface concentration, $C_{s}$, calculated according to eq. 6 . Here the diffusion coefficient for $\mathrm{HCl}$ in ice, $D_{i}$, is assumed to be $10^{-12}$ $\mathrm{cm}^{2} \mathrm{~s}^{-1}$. 


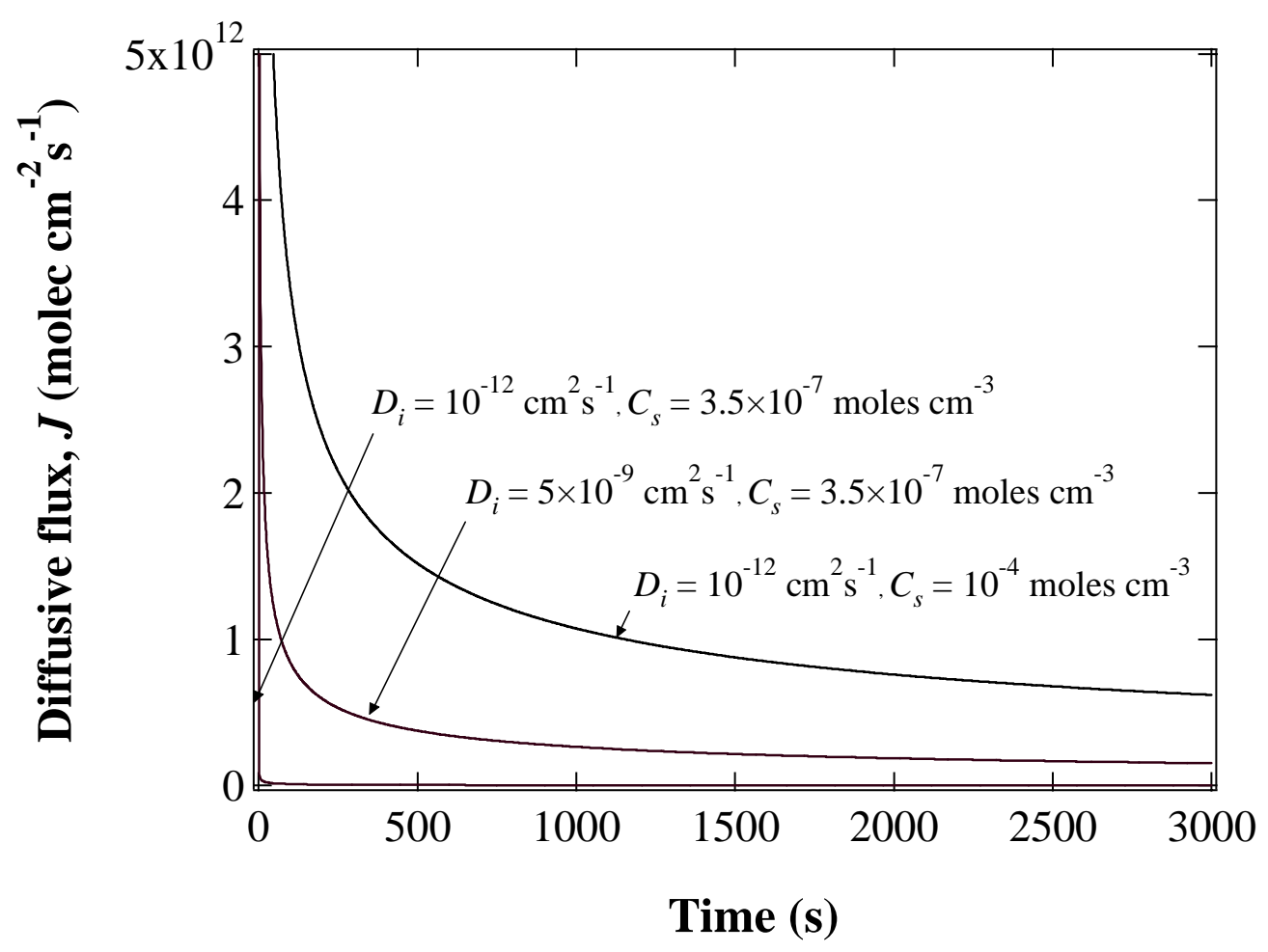

Figure S3. Diffusive flux , $J$, as calculated by eq. 7 for different values of the diffusion coefficient of $\mathrm{HCl}$ in ice, $D_{i}$, and the near-surface concentration, $C_{s}$.

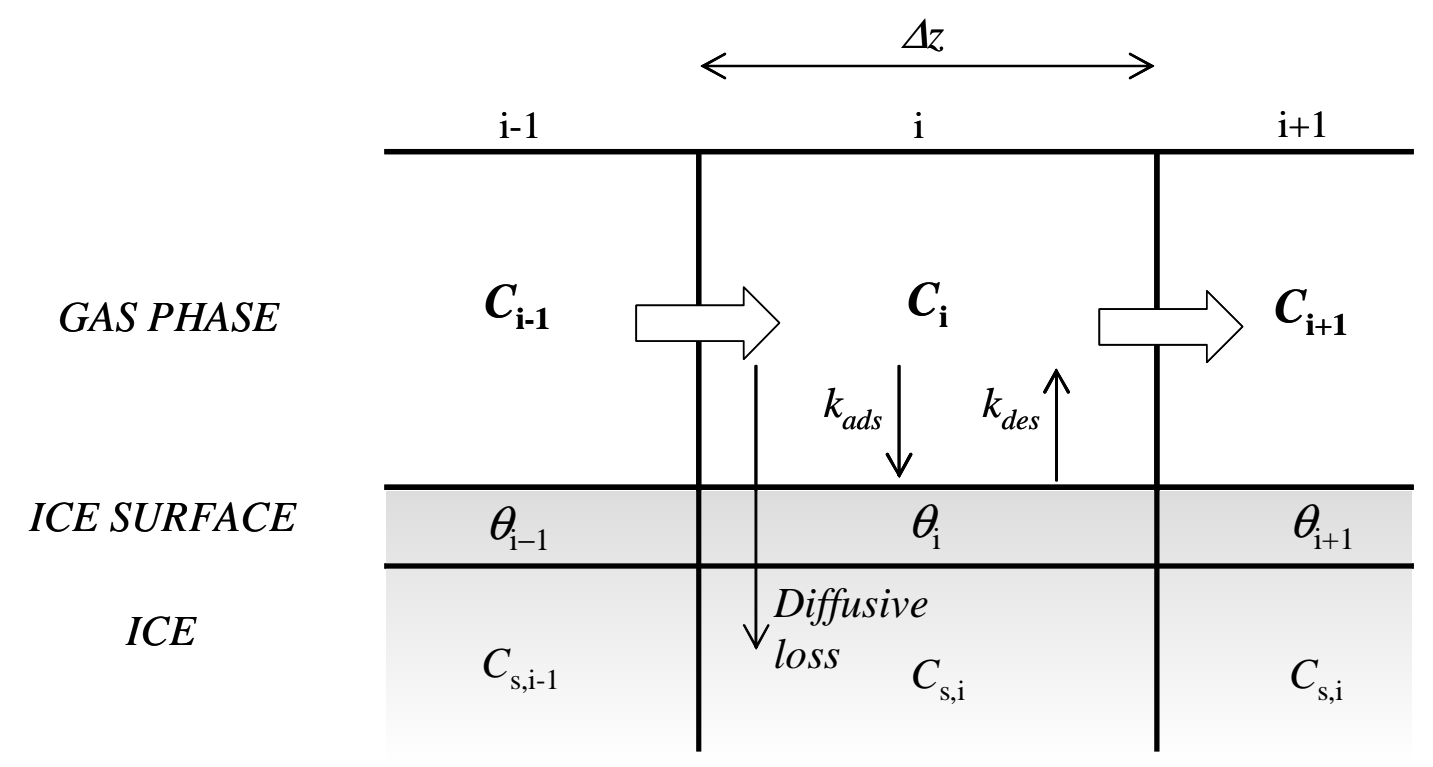

Figure S4. Schematic diagram for numerical modeling of flow tube with solute-wall interaction including diffusive loss. 


\section{References}

1. Abbatt, J. P. D. Geophys. Res. Lett. 1997, 24, 1479-1482.

2. Abbatt, J. P. D.; Beyer, K. D.; Fucaloro, A. F.; McMahon, J. R.; Wooldridge, P. J.; Zhang, R.; Molina, M. J. J. Geophys. Res. 1992, 97, 15819-15826.

3. Leu, M. T.; Keyser, L. F.; Timonen, R. S. J. Phys. Chem. B 1997, 101, 6259-6262.

4. McNeill, V. F.; Loerting, T.; Geiger, F. M.; Trout, B. L.; Molina, M. J. Proc. Natl. Acad. Sci. U.S.A. 2006, 103, 9422-9427.

5. Thibert, E.; Domine, F. J. Phys. Chem. B 1997, 101, 3554-3565.

6. Incropera, F. P.; DeWitt, D. P. Fundamentals of heat and mass transfer, 4th ed.; Wiley: New York, 1996.

7. Aguzzi, A.; Fluckiger, B.; Rossi, M. J. Phys. Chem. Chem. Phys. 2003, 5, 4157-4169.

8. Fluckiger, B.; Chaix, L.; Rossi, M. J. J. Phys. Chem. A 2003, 107, 2768.

9. Fluckiger, B.; Chaix, L.; Rossi, M. J. J. Phys. Chem. A 2000, 104, 11739-11750.

10. Heath, M. T. Scientific computing: an introductory survey, McGraw-Hill: New York, 1997. 